\title{
Lamp1 mediates lipid transport, but is dispensable for autophagy in Drosophila
}

Norin Chaudhry ${ }^{1+}$, Margaux Sica ${ }^{2+}$, Satya Surabhii ${ }^{2+}$, David Sanchez Hernandez ${ }^{2}$, Ana Mesquita $^{2}$, Adem Selimovic ${ }^{1}$, Ayesha Riaz ${ }^{1}$, Hua Bai ${ }^{3}$, Gustavo C. Macintosh ${ }^{1 *}$, and Andreas Jenny $\mathrm{y}^{2,4^{*}}$

${ }^{1}$ Roy J. Carver Dept. of Biochemistry, Biophysics and Molecular Biology, lowa State University, Ames, IA, USA

${ }^{2}$ Department of Developmental and Molecular Biology, ${ }^{4}$ Department of Genetics, Albert Einstein College of Medicine, New York, NY, USA

${ }^{3}$ Department of Genetics, Development and Cell Biology, lowa State University, Ames, IA, USA

${ }^{+}$Equal contribution; in alphabetical order.

*Corresponding authors: andreas.jenny@einsteinmed.org gustavo@iastate.edu 


\section{Abstract}

The endolysosomal system not only is an integral part of the cellular catabolic machinery that processes and recycles nutrients for synthesis of biomaterials, but also acts as signaling hub to sense and coordinate the energy state of cells with growth and differentiation. Lysosomal dysfunction adversely influences vesicular transport-dependent macromolecular degradation and thus causes serious problems for human health. In mammalian cells, loss of the lysosome associated membrane proteins LAMP1/2 strongly impacts autophagy and cholesterol trafficking. Here we show that the previously uncharacterized Drosophila Lamp1 is a bona fide homolog of vertebrate LAMP1/2. Surprisingly and in contrast to Lamp1/2 double mutant mice, Drosophila Lamp1 is not required for viability or autophagy, suggesting that autophagy defects in Lamp1/2 mutants may have indirect causes. However, Lamp1 deficiency results in an expansion of the acidic compartment in flies. Furthermore, we find that Lamp1 mutant larvae have defects in lipid metabolism as they show elevated levels of sterols and diacylglycerols (DAGs). Since DAGs are the main lipid species used for transport though the hemolymph (blood) in insects, our results indicate broader functions of Lamp1 in lipid transport. Our findings make Drosophila an ideal model to study the role of LAMP proteins in lipid assimilation without the confounding effects of their storage and without interfering with autophagic processes.

\section{Keywords:}

LAMP proteins; autophagy; Drosophila; lipid transport; lysosome

\section{Abbreviations:}

aa: amino acid; AL: autolysosome; APG: autophagosome; APGL: autophagolysosome; AV: autophagic vacuole (i.e. APG and APGL/AL); AVi: early/initial autophagic vacuoles; AVd: late/degradative autophagic vacuoles; Atg: autophagy-related; CMA: chaperone mediated autophagy; DAG: diacylglycerol; eMI: endosomal microautophagy; ESCRT: endosomal sorting complexes required for transport; FB: fat body; HDL: high density lipoprotein; LAMP: lysosome associated membrane protein; LD: lipid droplet; LDL: low density lipoprotein; Lpp: lipophorin; Ltp: Lipid transfer protein; LTR: Lysotracker; MA: macroautophagy; MEF: mouse embryonic fibroblast mTORC: mechanistic target of rapamycin complex; PV: parasitophorous vacuole; SNARE: soluble N-ethylmaleimide sensitive factor attachment protein receptor; Snap: synaptosomal-associated protein; st: starved; TAG: triacylglycerol; TEM: transmission electron microscopy; TFEB : transcription factor EB; TM: transmembrane domain; tub: tubulin; UTR: untranslated region. 


\section{Introduction}

Endocytosis, phagocytosis, and autophagy funnel proteins and lipids into lysosomes where they are degraded by hydrolases in their acidic environment [1-4]. Lysosomes, originally identified by DeDuve in the 1950s, were thus traditionally considered catabolic organelles degrading biomass to recycle metabolic building blocks for biosynthetic processes. Only more recently, the lysosomal surface has also been recognized as signaling hub regulating nutrient signaling and lysosomal biogenesis via recruitment of the nutrient sensor mTORC1 (mechanistic target of rapamycin) or the master regulator of lysosomal biosynthesis TFEB (transcription factor EB; reviewed in [5-8]). Lysosomal dysfunction affects membrane trafficking and repair on the one hand, and metabolism and signaling on the other [5-8] and thus has wideranging consequences for human health. Lysosomal storage diseases are caused by mutations in about 70 genes encoding hydrolases, lysosomal membrane proteins (LMPs), and transport proteins [8] and include Pompe disease caused by glycogen accumulation in lysosomes [9] and Nieman Pick Type $\mathrm{C}$ disorder resulting in lysosomal lipid, particularly cholesterol, accumulation [10]. Additionally, degradation of cytoplasmic proteins and organelles via autophagy is critically dependent on lysosomal function, and defective lysosomal proteolysis and autophagosomallysosomal fusion contribute to neurodegenerative diseases including Parkinson, Alzheimer's and Huntington disease $[3,11,12]$.

The LAMP (lysosome associated membrane protein) family of proteins are type 1 transmembrane domain proteins characterized by luminal LAMP domains stabilized by two S-S bridges (Fig. 1A) $[13,14]$ that are followed by a transmembrane domain and short C-terminus facing the cytosol and containing a $Y X X \Phi$ - type endosomal sorting signal (Fig. 1A) [15-17]. In vertebrates, LAMP1 and LAMP2 make up $~ 50 \%$ of proteins in the lysosomal membrane $[18,19]$. Their luminal domains are heavily $\mathrm{N}$ - and O-glycosylated and form a glycocalyx that is hypothesized to protect the lysosomal membrane from the acidic hydrolases [18]. While Lamp1 mutant mice are mostly normal [20], 50\% of Lamp2 mutants die within weeks of birth, and surviving animals show cardiomyopathy and an increase in autophagic vacuoles (AVs) in many tissues including the liver [21,22]. Importantly, human patients with mutations in LAMP2 have Danon disease, characterized by similar phenotypes [23,24]. LAMP2 encodes three spliceisoforms that differ in their TM region and C-termini. Lack of the $2 \mathrm{~B}$ isoform is sufficient to cause defects in macroautophagy (MA), the form of autophagy dependent on the formation of autophagosomes which engulf cytoplasmic content [21,22,24-26]. Arguably, the best characterized isoform is LAMP2A, which functions as substrate translocation channel in chaperone mediated autophagy (CMA), a form of autophagy specific for soluble cytoplasmic 
proteins containing a KFERQ-targeting motif. Lack of LAMP2A leads to premature aging-like phenotypes and enhances neurodegeneration [27-29]. Lamp1/2 double-mutant mice are embryonic lethal and double-mutant mouse embryonic fibroblasts (MEF) show a strong accumulation of autophagic vacuoles (AV) and unesterified cholesterol in late endosomes and lysosomes [2,18,25,30]. Furthermore, Lamp2 and Lamp1/2 double mutant MEFs and hiPSCderived cardiomyocytes from Danon disease patients revealed a block of MA flux [25,31]. However, the cause for the accumulation of $\mathrm{AVs}$ and the block of MA is not understood and could be indirect $[21,25]$.

In contrast to the two partially redundant mouse Lamp1/2 genes, the Drosophila melanogaster genome contains a single annotated Lamp1 gene evolutionarily related to vertebrate $L A M P 1 / 2$ that remains uncharacterized [32,33]. To our surprise, we find that Drosophila Lamp1 null mutants are homozygous viable and show no delay during development, suggesting normal steroid hormone signaling. Furthermore, Lamp1 mutants have no defect in macroautophagy or endosomal microautophagy (eMI), suggesting that LAMP proteins are not per se required for autophagy. However, we find a strong increase of acidic vesicles under basal and starvation conditions in the fat body that has functions akin to the mammalian liver. Furthermore, lipid analyses show that while levels of neutral fats (triacylglycerols; TAG) are unaffected in larvae, levels of sterols and medium chain diacylglycerols (DAG) are increased, denoting changes in lipid transport. Our results thus indicate that the roles of LAMP proteins in autophagy and in lipid homeostasis are likely independent processes.

\section{Results}

\section{Drosophila Lamp1 localizes to endolysosomes and is dispensable for viability}

Drosophila Lamp1 (CG3305) encodes a protein of 318 aa with a N-terminal signal peptide followed by a single LAMP domain with four conserved cysteines, which is $21 \%$ and $18 \%$ identical to the membrane proximal and $16 \%$ and $17 \%$ identical to the distal LAMP domain of human LAMP1 and LAMP2, respectively (Clustal 2.1) [34]. These characteristics suggest that it is the homolog of the mammalian LAMP1/2 genes (Fig. 1A) [32]. To determine the subcellular localization of Lamp1, we stained $3^{\text {rd }}$ instar larval fat bodies (FB) for Lamp1 with an antibody that we generated against its C-terminal peptide (Fig. 1A). Lamp1 localizes in a punctate pattern under fed and starved conditions (Fig. S1 E, G), a pattern that is specific, as no staining is found in Lamp $1^{6.1}$ mutants (Fig. S1F, H; see below for mutant). To determine the identity of Lamp1associated organelles, we labeled late endosomes and lysosomes of $3^{\text {rd }}$ instar larval FB using fluid phase endocytosis of fluorescent dextran (90 min chase period) $[35,36]$ followed by 
staining for Lamp1. Under fed and starved conditions Lamp1 strongly co-localizes with fluorescent dextran (Fig. 1D and $E$, respectively), also reflected in the quantification of Pearson's correlation coefficients of 0.6 and 0.7 , respectively (Fig. 1F) [37].

To address the function of Lamp1, we generated mutants using CRISPR-Cas9 [38] and obtained two independent alleles (Fig. 1B). Lamp1 $1^{6.1}$ contains frameshifts in exons two and three, well upstream of the TM domain, possibly encoding the first 90 aa of Lamp1, while in Lamp1 ${ }^{11 B}$, all but the first 8 aa are deleted (Figs. 1B and S1A). Additionally, the PiggyBac insertion Lamp1 $1^{e 879}$, inserted in the first intron of Lamp1, is an RNA null allele, as RT-PCR showed absence of any mRNA (Fig. S1B). Unexpectedly, all three Lamp1 alleles are homozygous viable and fertile and adults do not show an externally visible phenotype. In addition, Lamp $1^{6.1}$ mutants show no developmental delay, as they take a median of $140 \mathrm{~h}$ to pupation similar to WT controls (Fig. 1C).

Lamp1 protein migrates at an apparent $\mathrm{Mw}$ of $45 \mathrm{kDa}$ with additional larger bands of up to $60 \mathrm{kDa}$ in lysates of adult heads (Fig. S1C) or $3^{\text {rd }}$ instar larvae (Fig. S1D), thus considerably larger than its predicted $\mathrm{Mw}$ of $34.8 \mathrm{kDa}$, consistent with 11 predicted N-glycosylation sites upstream of a transmembrane domain (Fig. 1A). Importantly, these bands are specific to Lamp1, as they are absent from lysates of Lamp $1^{6.1}$ and Lamp $1^{11 B}$ mutants (Fig. S1C,D), also suggesting that all Lamp1 alleles are likely null alleles. Upon treatment of adult head lysates with Endo $\mathrm{H}$, which removes mannose rich oligosaccharides from proteins $[39,40]$, or PNGase $\mathrm{F}$ that removes all N-linked sugars [40], the apparent Mw of Lamp1 specific bands shifts towards smaller protein species at the expense of the largest ones, an effect that is stronger upon PNGase $\mathrm{F}$ treatment (Fig. 1G). The late endosome and lysosomal localization, and $\mathrm{N}$ glycosylation status of Lamp1 are consistent with it being a bona fide homolog of mammalian LAMP1/2.

\section{Expansion of the acidic endolysosomal compartment in Lamp1 mutants}

To assess changes in the endolysosomal compartment, we stained larval FB ex vivo with lysotracker LTR, that is retained in acidic structures upon protonation and has been used to monitor starvation induced MA as barely any Lysotracker positive structures are found under fed conditions [41]. Compared to control (Fig. 2A), FB of well-fed larvae of all three Lamp1 alleles show very pronounced increase in acidic structures (Fig. 2 B,C and Fig. S2; quantified in Fig. 2I). Importantly, this phenotype is rescued by a duplication that includes the Lamp1 locus (Lamp1 ${ }^{D p}$; Fig. 2D, quantified in I) and the phenotype is not restricted to larvae, but also found in FB of adult Lamp ${ }^{e 879}$ mutants (Fig. 2G,H; quantified in 2J). A similar, albeit smaller expansion of 
acidic structures is found upon starvation (Figs. 2E, F and S2D-F; quantified in 2I). The increase in LTR staining is not due to defects in the regulation of a starvation response in Lamp1 mutants, as the mRNA of Amyrel, the gene encoding an a-Amylase-like protein, known to be strongly induced by starvation [42,43], is low in fed larvae and normally upregulated by starvation to similar levels in WT and Lamp1 mutants (Fig. 2K).

To test if the LTR phenotype correlated with increased lysosomal activity, we first stained FB of $3^{\text {rd }}$ instar larvae with MagicRed, a Cathepsin B substrate that becomes fluorescent in active, acidic lysosomes $[44,45]$. Under basal, fed conditions and in contrast to increased LTR staining, Lamp1 mutants show no increase of Cathepsin B activity, suggestive of unchanged lysosomal activation (Fig. 3A-C; compare $w^{1118}$ with $\operatorname{Lamp}^{6.1}(\mathrm{~B})$ and $L a m p 1^{11 B}(\mathrm{C})$; quantified in $3 \mathrm{G}$ ). This is also supported by normal Acid Phosphatase activity in lysosome enriched fractions of Lamp1 mutant larval lysates (Fig. 3H), a common marker of lysosomal activity [46,47]. However, although quite variable, Cathepsin B activity is higher in Lamp1 mutants under starved conditions (Fig. 3D-F; quantified in $3 G$ ), suggesting that lysosomal activation can be abnormal under certain stress conditions.

\section{Lamp1 is dispensable for autophagy}

The expanded acidic compartment could be suggestive of a MA defect. MA is well characterized in $3^{\text {rd }}$ instar fat body $[36,48,49]$ and autophagic flux is commonly assessed using a tandem-tagged GFP-mCherry-Atg8a reporter which fluoresces green and red in autophagosomes, but red only in autolysosomes due to GFP fluorescence being quenched by their acidic $\mathrm{pH}$ [50]. However, neither under fed (Fig. $4 \mathrm{~B}$; quantified in 4E) nor starved conditions (Fig. 4D; quantified in 4E) do Lamp1 $1^{6.1} /$ Lamp $^{11 B}$ transheterozygous or Lamp $1^{6.1}$ homozygous mutants (quantified in Fig. 4E) show a difference in APGs (green puncta) nor total autophagic structures (red puncta) compared to controls (Fig. 4A, B; quantified in 4E). Importantly, we find a robust, normal stimulation of MA upon starvation as seen in the strong increase of autolysosomes and a tendency towards an increase in APGs (Fig. 4C, D; quantified in Fig. 4E). Prior to fusion of APGs with lysosomes, APGs recruit the SNAREs Syx17 (STX17 in vertebrates) and Snap29 as part of the fusion process [51,52]. Consistent with functional starvation induced MA, Snap29 is normally recruited to autophagosomes marked by mCherryAtg8a of $3^{\text {rd }}$ instar FB cells of Lamp $1^{6.1}$ homozygous mutants (quantified in Fig. $4 \mathrm{H}$ ) or Lamp $1^{6.1}$ / Lamp $1^{11 B}$ transheterozygotes (Fig. 4F,G; quantification in $4 \mathrm{H}$ ). In addition, there is no change in the expression of the core MA genes Atg5 and Atg8a in adult flies (Fig. 4E). Our data thus 
suggest, that, in contrast to humans and mice [21-24], there is no basal or starvation induced MA defect in the absence of Lamp1 in Drosophila.

In mammals, in addition to MA, CMA and eMI also contribute to autophagic protein degradation [1-4]. eMI has recently been identified in flies based on its selective requirement of Hsc70-4 and the ESCRT machinery [53,54]. On the other hand, to date, CMA has only been shown to occur in mammals and fish and other species (except for birds) lack amino acids critical for Hsc70/HSPA8 interaction in their LAMP proteins [28,55]. We therefore tested if Lamp1 is required for eMI, but found that similar to MA, there is no eMl defect in Lamp1 mutants, as prolonged starvation normally induces eMI sensor puncta (KFERQ-PAmCherry) in Lamp $1^{6.1}$ mutants (Fig. 5; compare heterozygous larval FB (A,C) with homozygous mutant tissue $(B, D)$; quantification in $5 E)$. Given the essential role of LAMP2A in CMA and that fact that KFERQ-PAmCherry acts as CMA sensor in vertebrates [56,57], the lack of differences in the levels of KFERQ-mCherry puncta between wild-type and Lamp1 mutants support the idea that flies lack a CMA-like process.

To independently assess MA, we performed morphometric analyses of autophagic structures on TEM sections of fed and starved Lamp $1^{6.1}$ mutant $3^{\text {rd }}$ instar FB (Fig. 6). No difference between wild-type and Lamp1 mutant tissue was found for the density of mature autolysosomes (examples in Fig. 6A, C; quantified in Fig. 6G; see materials and methods for definition of structures) and lysosomes (examples in Fig. 6D, E; quantified in Fig. 6H). A tendency towards an increased area of APGLs was found for fed Lamp1 $1^{6.1}$ mutant FB, while the size distribution of those structures changed towards smaller ones under starvation (Fig. 6l). Autophagosomes (AVi in e.g. [18]) were very rare in all cases. Overall, our data thus show no indication for autophagy defects in Lamp1 mutants.

\section{Altered lipid content in Lamp1 mutants}

Lamp1/2 mutant MEFs accumulate cholesterol in the endolysosomal system and it was shown that LAMP1/2 proteins bind cholesterol for storage in lysosomes [26,58]. We therefore determined whether lack of Lamp1 altered lipid profiles of mutant $3^{\text {rd }}$ instar larvae. Lamp $1^{\text {e879 }}$ mutants showed elevated total sterol levels, a phenotype rescued by a Lamp1 duplication (Fig. 7A). In flies, dietary lipids are taken up by enterocytes in the gut and converted to diacylglycerols (DAG), secreted and transported in the hemolymph (blood) as lipoprotein complexes, from where they are taken up by other tissues such as FB cells that convert them to triacyclglycerols (TAGs) for storage [59]. No changes were found in triglycerides in Lamp1 fe879 $^{\text {eng }}$ mutants (Fig. 7B), consistent with no change in lipid droplet density (LD) in the fat body in our 
TEM analyses (Fig. S3A). In contrast, Lamp1 mutant larvae show elevated levels of DAGs that are rescued by Lamp1 duplication (Fig. 7C). The increase is specific for DAGs with medium chain fatty acid tails (Fig. 7C), characteristic of DAGs associated with lipoproteins, which suggests that lipid transport or mobilization is affected. No changes were found on glycogen density by TEM (Fig. S3C).

\section{Discussion}

Our assessment of the function of Lamp1 using a marker of fluid phase endocytosis chased into lysosomes in living FB cells showed that Lamp1 localizes to late endosomes and lysosomes (Fig. 1), consistent with it being the homolog of the mammalian LAMP1/2 proteins, which is also supported by gene synteny of the Lamp1 locus with vertebrates (Dr. L. Lescat and AJ; unpublished observation). Unexpectedly, Lamp1 mutants are homozygous viable and show no developmental delay, distinct from mice lacking both, partially redundant Lamp1/2 genes, which die around E15.5 [26]. We found a large expansion of the cellular acidic compartment under basal conditions and starvation in Lamp1 mutants. Again, this is distinct from mouse Lamp2 mutants and differentiated cardiomyocytes derived from hiPSC of Danon patients, in which no change in LTR staining was observed [31]. It is worth mentioning that lysosomal $\mathrm{pH}$ is driven by a difference in membrane potential between the cytoplasm and the lysosomal lumen (more positive), which could be altered in Lamp1 mutants [60]. Interestingly, under basal conditions, the increase in acidic structures does not result in increased activity of lysosomal hydrolases, as we saw no increase in Cathepsin B or Acid Phosphatase activity (Fig. 3). Expanded acidic structures have been reported upon knockdown of Drosophila Gga (Golgilocalized, $\mathrm{y}$-adaptin ear containing, ARF binding protein) which is required for the transport of Cathepsins to lysosomes and thus for their proteolytic processing and activation. However, we found no indication of altered Cathepsin L processing in FB of Lamp1 mutants (not shown), and the increase in LTR-positive puncta could thus represent an increase in late endosomes, as these organelles are acidified but lack hydrolytic activities $[61,62]$.

Lamp2 and Lamp1/2 double mutant cells have a block in autophagic flux as shown by measurements of LC3-II levels [31] and of APGs using tandem GFP-RFP-LC3 reporters [25,31] and EM [22]. However, the mechanistic reason for this phenotype is unclear. It was suggested that LAMP2 is required for APG-Iysosome fusion [25], or to promote fusion via simultaneous interaction with Atg14 on APGs and the lysosomal SNARE Vamp8 [31], therefore having a direct function in fusion. However, Hubert et al. [25] showed failure of APGs to recruit the SNAREs STX17 and SNAP29 required for fusion of APGs to lysosomes in Lamp2 and Lamp1/2 
double mutant cells, which is not easily reconcilable with a direct function of LAMPs in MA. Additionally, non-functional vesicular transport and thus a potentially indirect effect has also been suggested as reason for defects in phagosome-lysosome fusion in Lamp1/2 mutant MEFs [63]. Furthermore, the initial TEM analysis of Lamp1/2 double mutant MEFs found no defect in the formation of APGs or their fusion with lysosomes, but rather a retardation in maturation of autolysosomes [26]. Our results show that basal MA flux and starvation induced MA are normal in Drosophila Lamp1 mutant fat body (Fig. 4A-E). Consistently, we find normal recruitment of Snap29 to APGs (Fig. $4 \mathrm{~F}-\mathrm{H}$ ) and no accumulation of APGs in our TEM analyses, arguing against an essential direct function of LAMPs in MA. Alternatively, a more direct function of LAMPs in MA could have evolved after separation of vertebrates from arthropods. Furthermore, we cannot rule out that Lamp1 affects MA elicited by other forms of cellular stress. Importantly, we also show that, as in mammals [64,65], Lamp1 is dispensable for eMI and, since our eMI sensor is equivalent to the mammalian CMA sensor [53,57], our data functionally confirm that there is no evidence for CMA in Drosophila.

LAMP proteins have also been implicated in lipid metabolism [66]. LAMP1 and LAMP2 can bind cholesterol [58], and Lamp1/2 double mutants accumulate cholesterol in the endolysosomal system [26,67]; thus LAMP proteins have been implicated in the handover of cholesterol to the NPC lysosomal cholesterol export system [58]. Consistent with those findings, Drosophila Lamp1 mutant larvae also have increased sterols levels, although the increase is small compared with the effect observed in mice. Fruit flies are sterol auxotrophs and rely on dietary sterols for survival [68], and, consistently, both yeast-derived and plant-derived sterols were identified in our analysis (not shown).

Importantly, Lamp1 mutant larvae also displayed a significant increase in DAGs. Unlike vertebrates, insects use DAGs as the main lipid species for transport of dietary lipids from the midgut to other tissues [59,69]. Lipids from the diet are digested by secreted lipases and esterases in the midgut, followed by absorption of fatty acids, sterols, and other molecules by enterocytes [59,69]. These cells then synthesize DAGs for export into the hemolymph, which transports lipids as lipoprotein complexes to other tissues [69]. Lipophorin (Lpp), a member of the ApoB family, is the main hemolymph lipid carrier [70]. The most abundant lipids in Lppcontaining lipoproteins are DAGs (70\%), and phosphatidylethanolamine $(20 \%)$, while sterols make up only 5\% [70]. ApoLpp is synthesized in the FB and released to the hemolymph as high-density lipoproteins (HDL) that are loaded with phospholipids but lack DAGs. HDLs travel to the gut where they are loaded with DAGs with the help of another lipoprotein particle 
containing lipid transfer protein (LTP) and become low-density lipoproteins (LDL) [69,70]. LDLs are transported in the hemolymph for final use in organs $[59,69]$.

Importantly, transported DAGs contain medium chain fatty acid, with a large proportion of C12 and C14 tails [70,71], corresponding to the species showing the most significant increase in Lamp1 mutants (combined chain lengths of 24 and 28 carbons; Fig. 7). Thus, it is apparent that Lamp1 has a broader role in lipid assimilation and is not limited to sterol metabolism. However, the mechanism by which Lamp1 affects DAGs is less clear. Given Lamp1's prevalent localization in late endosomes and lysosomes, it can be hypothesized that the protein has a role in endocytosis, and lack of Lamp1 could affect endocytosis-dependent lipid transport. For example, it was shown that Lpp loading partially depends on endocytosis, as the intermediary lipoprotein LTP needs to be internalized in midgut enterocytes for loading. In turn, LTP is necessary for loading of DAGs and sterols into Lpp for transport via the hemolymph $[59,70]$. Moreover, LTP RNAi results in large accumulation of medium chain DAGs in the gut [70] and defective LTP endocytosis in Lamp1 mutants could explain the observed lipid phenotype.

Defects in phagocytosis have been previously reported in LAMP1/2 mutant mice fibroblasts transfected with FcyllA receptors [63]. In this case, phagosomes acquire Rab5 but not Rab7, and fail to fuse with lysosomes, indicating that endocytosis is impaired at a late step in the process. It was proposed that the endocytosis defect is associated with a failure of endosomes and lysosomes to move on microtubules [63]. Similarly, defective compensatory endocytosis was observed in $L A M P 1 / 2$ mutant fibroblast during plasma membrane repair [72]. The reduced ability of $L A M P 1 / 2$ mutant fibroblasts to carry out compensatory endocytosis also explains their increased resistance to Trypanosoma cruzi infection, as the parasite causes plasma membrane injuries and then hijacks the compensatory endocytosis process to enter the host cell $[72,73]$. However, in this case it was proposed that the role of LAMP2 in endocytosis may be indirect, as a decrease in cholesterol level in the plasma membrane associated with failure to recruit caveolin-1 could have led to a reduction in endocytosis [72]. Similarly, liver-stage Plasmodium berghei parasites have reduced growth in LAMP1/2 mutant host cells because the parasite fails to recruit late endosomes and lysosomes to the parasitophorous vacuole (PV) [74]. The same phenotype was observed for NPC mutant host cells or when host cells were treated with the amphipathic steroid 3-b-[2-(diethylamine)eth-oxy]androst-5-en-17-one (U18666A). The common phenotype in the three cases is the accumulation of cholesterol in late endosome/lysosomes, suggesting that sequestration of cholesterol and potentially reduction of the available sterol pool leads to the defects in late endosome and lysosome recruitment to the PV, and/or reduced availability of sterols necessary for parasite growth [74]. These results further support the 
hypothesis that lack of LAMP proteins results in endocytosis defects, although the direct participation of LAMP proteins in specific membrane fusion events, or their indirect effect through changes in lipid composition of the membranes needs to be resolved. In any case, the disruption of endocytosis in Lamp1 mutants could not completely block this process, since manipulation of Drosophila genes essential for endocytosis also disrupt lysosome biogenesis and result in an overall decrease in LTR-positive puncta [75], opposite to the phenotype observed for Lamp1 mutants.

Our analysis indicated that Drosophila Lamp1 is necessary to maintain the normal cellular composition of acidic organelles, and for lipid transport. Our data showing that basal and starvation induced autophagy is not affected in Lamp1 mutants, while sterol and DAG levels changed thus lend support to the previous suggestion that changes in sterol levels and the defects in autophagic flux observed in Lamp1/2 mutant mice are independent phenotypes [67]. Moreover, the use of DAGs as the main species for lipid transport in insects rather than TAGs as in vertebrates allowed us to identify a broader effect for LAMP proteins in lipid metabolism without a confounding effect of lipid storage. These characteristics make Drosophila an ideal model to further dissect the roles of LAMPs in lipid metabolism and organelle traffic.

\section{Materials and Methods}

\section{Fly strains and genetics.}

All fly stocks were maintained at $25 \square^{\circ} \mathrm{C}$ and reared on standard cornmeal media unless otherwise indicated. PBac\{RB\}Lamp $1^{e 00879}$ (here $L a m p 1^{e 879}$ ) is a piggyBac insertion [76] in the first intron of Lamp1 (Fig. 1B) and is a RNA null allele (Fig. S1D). y1 M\{nos-Cas9.P\}ZH-2A w* (BL\# 54591) was from the Bloomington stock center. $\mathrm{Dp}(2 ; 3) P 6-B 2^{[L a m p 1]}$ is a duplication including the Lamp1 locus in VK31 on chromosome 3L (referred to here as Dp) that was obtained from Genetivision (Huston, TX). UASp-GFP-mCherry Atg8a u2.6 (Chr. II; tandem tag) was from Dr. I. Nezis (U. Warwick, UK) and was recombined onto the Lamp ${ }^{6.1}$ chromosome. Lamp 1 ${ }^{6.1}$; r4-Gal4 and Lamp1 11B; r4-Gal4 used in the flux assays are based on r4-Gal4 obtained from Dr. T. Neufeld (U. Minnesota)[77], who also provided UAS-mCherry-Atg8a lines [78]. UASKFERQ-PAmCherry 3B was as in [53]. See Table S1 for exact genotypes.

To measure pupation timing, flies were allowed to lay eggs overnight onto grape juice plates before removing hatched larvae by floating them on $20 \%$ sucrose for embryo synchronization. After verification of the absence of larvae, plates were incubated for $2 \mathrm{~h}$ at $25{ }^{\circ} \mathrm{C}$ and newly hatched larvae were floated off with $20 \%$ sucrose, collected, and placed at roughly 30 
larvae/vial onto regular food ( $t=25 \pm 1 \mathrm{~h}$ to account for $24 \mathrm{~h}$ of embryogenesis). Pupating larvae were scored every $8 \mathrm{~h}$.

\section{Molecular biology and transgenic flies}

Transgenes were injected by Rainbow Transgenic flies (CA). pCFD4_Lamp_13 pCFD4_lamp1-30_4-4 were made by Gibson assembly (NEB \# E5510S) using oligos Lamp1_crips_61_for, Lamp1_crisp_63_rev and Lamp1 exon 4_4, Lamp1 exon_1_30, respectively (Table S2) as described [38]. Transgenic lines were crossed with isogenized nosCas9/FM6; Frt40/CyO, allowing generation of Lamp $1^{6.1}$ directly on a FRT40 chromosome. Genotyping oligos are indicated in Table S1. Lamp1 ${ }^{6.1}$ FRT40 (Lamp1 $1^{6.1}$ in text) contains frame shifts in exons 2 (5 bp deletion) and exon 3 (21 bp at expense of deletion of $8 \mathrm{bp}$, thus a net insertion of $13 \mathrm{bp}$ ) resulting in a predicted protein truncated after 90 aa of Lamp1 and an additional ectopic 32 residues (Figs. $1 \mathrm{~B}$ and $\mathrm{S} 1 \mathrm{~A}$ ). Lamp $1^{11 B}$ deletes all but the first 8 aa of the Lamp1 ORF including the stop codon (3067 bp, 1 bp insertion; 3066 bp net deletion; Figs. 1B and S1A). Lamp1 $1^{6.1}$ FRT40 was outcrossed 6 times against $w^{1118}$ to lose an off-target lethal mutation. In both cases, non-targeted chromosomes were exchanged.

For qPCR, total RNA was extracted using TRIzol reagent (Thermo Fisher Scientific, Waltham, MA, USA) from 25-30 larvae or whole adult flies, DNase-treated (Turbo DNA-free kit; Ambion) and reverse transcribed using SuperScript III First Strand synthesis kit (Invitrogen). qPCR was performed in triplicates with an ABI prism 7300 Sequence Detection System (Thermo Fischer Scientific, Waltham, MA, USA) using Absolut qPCR with SYBR Green + Rox kit (Thermo Fischer Scientific; see Table S2 for primers). mRNA abundance was normalized with $R p L 32$ as housekeeping control using the $\Delta \Delta \mathrm{C}_{\mathrm{T}}$ method [79].

\section{Biochemistry and lipid analyses}

For Western blot analyses, larvae were starved for $4 \mathrm{~h}$ on $20 \%$ sucrose and adults for $24 \mathrm{~h}$ with water saturated filter papers. 10 fly heads or 20 washed larvae were suspended in $50 \mu \mathrm{l}$ or $200 \mu \mathrm{l}$ 1x Laemmli buffer with 2\% SDS (2\% SDS, 10\% glycerol, 5\% 2-mercaptoethanol, 0.002\% bromophenol blue and $62.5 \mathrm{mM}$ Tris $\mathrm{HCl}, \mathrm{pH} 6.8$ ), respectively, and boiled for $5 \mathrm{~min}$ at $95{ }^{\circ} \mathrm{C}$. After homogenization with a motor pestle, lysates were boiled again and centrifuged twice for 10 $\min$ at $20,000 \mathrm{~g}$ at room temperature. After each centrifugation, the $90 \%$ of the liquid phase was removed avoiding lipids floating on top. Upon separation of the proteins by SDS-PAGE, proteins were transferred to nitrocellulose membranes and probed with NY2403 anti Lamp1 (see below) at 1:5000 and anti aTubulin (Sigma, T5168; 1:10,000) as loading control. 
For deglycosylation assays, 40 adult heads of indicated genotypes were lysed in 200 $\mu$ RIPA buffer (150 mM NaCl, 5 mM EDTA pH 8.0, 20 mM Tris pH 8.0, 1\% NP40, $0.25 \%$ Deoxycholate) by homogenization with a motor pestle. After clearing at $10,000 \mathrm{~g}$ for $10 \mathrm{~min}$ at $4^{\circ} \mathrm{C}, 70 \mu \mathrm{g}$ protein (as determined with a Lowry assay) was combined with $3 \mu$ denaturing buffer (5\% SDS, $400 \mathrm{mM} \mathrm{DTT}$ ), heated to $95^{\circ} \mathrm{C}$ for $10 \mathrm{~min}$ prior to addition of protease inhibitors. $2 \mu \mathrm{l}$ Endo $\mathrm{H}$ (NEB P0702S) and $3 \mu$ of G3 reaction buffer (NEB, MA) and $2 \mu$ PNGase F (NEB P0704S), 3 $\mu l$ $10 \%$ NP40, and $3 \mu \mathrm{l}$ G2 reaction buffer were added, respectively. After incubation for $18 \mathrm{~h}$ at $37^{\circ} \mathrm{C}$, samples were boiled in Laemmli buffer and processed for Western blot analysis.

To measure Acid Phosphatase activity, a fraction containing lysosomes, mitochondria, and cytoplasm was prepared by centrifugation from 15 to $303^{\text {rd }}$ instar larvae as described [46], with the addition of cOmplete, Mini, EDTA-free Protease Inhibitor Cocktail (Sigma) in the extraction buffer. The presence of lysosomes in this fraction was confirmed by Lysotracker staining and observation under fluorescence microscope. Protein concentration was determined using the Pierce BCA protein assay kit (Thermo Fisher Scientific). Acid phosphatase activity was determined fluorometrically using 4-methylumbelliferyl phosphate as substrate [80].

For lipid quantification, ten $3^{\text {rd }}$ instar larvae were weighed and homogenized with $350 \mu$ of hot methanol $\left(60^{\circ} \mathrm{C}\right)$, spiked with $25 \mu \mathrm{g}$ of ribitol and $25 \mu \mathrm{g}$ of nonadecanoic acid as internal standards. The mixture was immediately incubated at $60^{\circ} \mathrm{C}$ for $10 \mathrm{~min}$ and sonicated for $10 \mathrm{~min}$. Samples were then extracted with $350 \mu \mathrm{l}$ of chloroform and $300 \mu \mathrm{l}$ of water and centrifuged for 7 minutes at $14,000 \mathrm{~g}$. The lower, non-polar fraction was transferred to new vials and dried in a Speed-Vac concentrator. Non-targeted metabolite analysis was used to quantify sterols and diacylglycerols (DAG). The samples were methoximylated and silylated as described[81]. One microliter of the derivatized samples was injected into an Agilent $6890 \mathrm{GC}$ interfaced to an Agilent 5973 quadrupole MS with a HP-5ms (5\%-Phenyl)-methylpolysiloxane column (30 m x $0.25 \mathrm{~mm} \times 0.25 \mu \mathrm{m}$, Agilent) in splitless mode. The temperature was programmed from 70 to $320^{\circ} \mathrm{C}$ at $5^{\circ} \mathrm{C} / \mathrm{min}$ with helium flow rate at $1.0 \mathrm{~mL} / \mathrm{min}$ and inlet temperature at $280^{\circ} \mathrm{C}$. El-MS ionization energy was set to $70 \mathrm{eV}$ and the interface temperature was $280^{\circ} \mathrm{C}$. The GC-MS data files were deconvoluted and searched against an in-house MS-library and the NIST 14 Mass Spectral Library using NIST AMDIS software [82]. Triacylglycerols (TAG) were analyzed with an Agilent Technologies 1200 Series four solvent gradient capable HPLC coupled to Agilent 1200 series evaporative Light scattering detector (ELSD) and a Supelco Ascentis-Si $(25 \mathrm{~cm} \times 2.1 \mathrm{~mm}$ x5 um) column. Elution was performed using mobile phases containing $A$ : hexane with $1 \%$ isopropanol with $0.4 \%$ acetic acid added, and $B$ : $100 \%$ isopropanol. The solvent gradient used was: $0-5$ min $100 \%$ solvent $A, 5-10$ min gradient to $95 \%$ solvent $A, 10-15 \min 95 \%$ solvent $A$, 
15-20 $\min 100 \%$ solvent $A$; with a flow rate of $0.8 \mathrm{ml} / \mathrm{min}$. ELSD: Nitrogen flow was 2.1 with $110^{\circ} \mathrm{C}$ source temperature. A mixture of TAGs $(2 \mathrm{mg} / \mathrm{ml})$ was used as quantification standard. All lipids were normalized using the dry weight after extraction.

\section{Autophagy, Lysotracker, Magic Red, and Dextran assays}

Dextran uptake assays to label lysosomes was performed as described [35,36]. Briefly, fed or starved larvae were washed, inverted and incubated with fluoro-Ruby-Dextran (1:100 of 100 $\mathrm{mg} / \mathrm{ml}$ stock; 10,000 MW; Invitrogen CA) in Graces medium for $10 \mathrm{~min}$, followed by 6 washes and a 90 min chase to allow the Dextran to be transported to lysosomes. Fixation and staining was as described below. Co-localization was calculated using Pearson's correlation coefficient, turning one channel by $180^{\circ}$ as control [37].

MA flux was determined using a tandem GFP-RFP-Atg8a reporter as described [50]. Briefly, well-fed mid $3^{\text {rd }}$ instar larvae of appropriate genotypes were washed with $\mathrm{H}_{2} \mathrm{O}$ and incubated for 3-4 $\mathrm{h}$ in $35 \mathrm{~mm}$ petri dishes with 3 Whatman filter paper soaked in $800 \mu \mathrm{l} 20 \%$ sucrose supplemented with heat-inactivate yeast (fed) or $20 \%$ sucrose only (for starvation) [48]. After dissection and fixation, FB lobes of approximately 5 larvae were mounted in $20 \mu \mathrm{DAPI}$ fluoromount-G (Southern Biotech 0100-20) per slide and imaged on an ApoTome.2 system using an Axiovert 200 equipped with a 63x 1.4 NA oil lens (Carl-Zeiss, Oberkochen, Germany). Quantification was done using the atgCOUNTER script in Fiji to threshold and exclude nuclear signal [50].

eMI activity was determined as described[53] after recombining r4-Gal4 with UAS-KFERQPAmCherry $3 B$ (once in Lamp $1^{6.1}$ background, the strain was backcrossed to homozygous Lamp $1^{6.1}$ flies to consistently have only one copy of the reporter construct). FB was imaged as above.

For Lysotracker staining, $3^{\text {rd }}$ instar larvae were starved in PBS for $4 \mathrm{~h}$ (Fig. 2) or $20 \%$ sucrose (Fig. S2). Larval or adult FB Larval and adult flies fat body tissues were dissected in 1X PBS, incubated for 3 to 5 min in $100 \mu \mathrm{M}$ Lysotracker- Red DND-99 (Life Technologies, Carlsbad, CA, USA)[49] and $1 \mu \mathrm{M}$ Höchst 33342 (Thermo Fisher Scientific Inc, Rockford, IL, USA) in PBS and immediately imaged using a Zeiss Imager (larval FB) or an Olympus BX51WI (adult tissues) using identical conditions across genotypes. FB cells that contained any LTR-positive puncta in adult flies or FB cells that contained more than 5 LTR-positive puncta in larvae were scored as positive.

For Magic red staining (Cathepsin- B Assay Kit; Immunochemistry Technologies LLC, Bloomington, MN, USA), larvae were starved for $4 \mathrm{~h}$ in $20 \%$ sucrose (supplemented with heat 
inactivated yeast for fed controls), washed and dissected in Graces medium (Invitrogen, CA; without serum in case of starved larvae). Larvae were inverted in the corresponding Graces medium and stained for $10 \mathrm{~min}$ in the same medium supplemented with Magic red substrate (total dilution 1:150) and DAPI (14.3 M stock at 1:2000). After 2 rinses in corresponding Graces medium, FB was dissected on glass slides and immediately imaged on a Zeiss ApoTome with a 40x/1.3NA oil DIC lens.

\section{Immunohistochemistry and EM analyses}

Lamp1 antibodies were generated against $\mathrm{KLH}$ coupled C-terminal peptide (CARRRSTSRGYMSF) in rabbits (\#NY2403) by Covance (Denver, PA; animal protocol \# 20170902) and affinity purified for use in immunohistochemistry (1:400-1:1000). Staining of FB was done as described [53,83]. Rabbit anti Snap29 [51] was used at 1:1000. Secondary antibody Alexa488 anti-rabbit (A11034; Invitrogen, CA) was used at 1:300.

For TEM, larvae were treated and processed as above, and, after inversion, fixed with $2.0 \%$ paraformaldehyde, $2.5 \%$ glutaraldehyde in $0.1 \mathrm{M}$ sodium cacodylate buffer, postfixed with $1 \%$ osmium tetroxide followed by $2 \%$ uranyl acetate, dehydrated through a graded series of ethanol and embedded in LX112 resin (LADD Research Industries, Burlington VT). Ultrathin sections were cut on a Leica Ultracut UC7, stained with uranyl acetate followed by lead citrate and viewed on a JEOL 1200EX transmission electron microscope at 80kv. Morphometric quantification was done by outlining indicated structures in Fiji/lmageJ and tabulating their area [84] on blindly and randomly selected fields of view (excluding only nuclei). APGLs were identified as electron dense (dark) structures with very well or less-well (likely more degrading already) defined content, in contrast to ALs that were less electron dense (more similar to surrounding cytoplasm) and contained ill-defined content (advanced degradation). APGLs and ALs are likely similar to AVds in e.g. [18]. Lysosomes (L) are very electron dense without halo (distinguishing them from peroxisomes) and small. Structure density (are per field of view of 100 $\mu \mathrm{m}^{2}$ ) or size distribution was analyzed using the Mann-Whitney and Kolmogorov-Smirnov tests.

\section{Statistical analysis}

Statistical tests were done with GraphPad Prism (Versions 8\&9; GraphPad Software, La Jolla, CA) using indicated tests and corrections. Unless noted, graphs represent means with standard error of means (SEM). ' $n=$ ' indicates fields of view unless indicated. As larvae are bilaterally symmetric and have two main FB lobes, we cannot exclude that two fragments may 
have originally belonged to the same lobe in the preparation. It is therefore a reasonable estimate that the number of animals is minimally about $n / 2$ and maximally equal to $n$.

\section{Acknowledgements}

We thank Drs. T. Neufeld, I. Nezis, G. Juhasz, T. Vaccari, and the Bloomington Drosophila Stock centers for kindly sharing fly strains. We are grateful to Dr. T. Vaccari for antibodies and to Drs. Mimi Kim (Division of Biostatistics, Einstein) and Ana Maria Cuervo for advice on the analyses of TEM images. We thank Dr. L. Ambrosio for help with fly genetics, the W. M. Keck Metabolomics Research Laboratory at lowa State University for help with lipidomics analyses, and Drs. J. Secombe and A. Melendez for comments on this manuscript and the Einstein Analytical Imaging Facility (NIH P30CA013330) for support. This work was supported by AHA postdoctoral fellowship 18POST34030231 (to A.M), NIH/NIGMS grant GM119160 (to A.J.), and grants of the Roy J. Carver Charitable Foundation (Muscatine, lowa) and NSF grant MCB1714996 (to G.C.M.). A.R and N.C. were further supported by Fulbright fellowships.

\section{Declaration of interest statement}

The authors declare no conflict of interest. 


\section{Literature Cited}

1. Herb M, Gluschko A, Schramm M. LC3-associated phagocytosis - The highway to hell for phagocytosed microbes. Seminars in cell \& developmental biology. 2020 May;101:68-76.

2. Kaushik S, Cuervo AM. Chaperone-mediated autophagy: a unique way to enter the lysosome world [Research Support, N.I.H., Extramural

Research Support, Non-U.S. Gov't

Review]. Trends Cell Biol. 2012 Aug;22(8):407-17.

3. Kaushik S, Cuervo AM. Proteostasis and aging. Nat Med. 2015 Dec;21(12):1406-15.

4. Pryor PR, Luzio JP. Delivery of endocytosed membrane proteins to the lysosome. Biochim Biophys Acta. 2009 Apr;1793(4):615-24.

5. Ballabio A, Bonifacino JS. Lysosomes as dynamic regulators of cell and organismal homeostasis. Nat Rev Mol Cell Biol. 2020 Feb;21(2):101-118.

6. Savini M, Zhao Q, Wang MC. Lysosomes: Signaling Hubs for Metabolic Sensing and Longevity. Trends Cell Biol. 2019 Nov;29(11):876-887.

7. Shin HR, Zoncu R. The Lysosome at the Intersection of Cellular Growth and Destruction. Dev Cell. 2020 Jul 20;54(2):226-238.

8. Trivedi PC, Bartlett JJ, Pulinilkunnil T. Lysosomal Biology and Function: Modern View of Cellular Debris Bin. Cells. 2020 May 4;9(5).

9. Kohler L, Puertollano R, Raben N. Pompe Disease: From Basic Science to Therapy. Neurotherapeutics. 2018 Oct;15(4):928-942.

10. Lloyd-Evans E, Platt FM. Lipids on trial: the search for the offending metabolite in Niemann-Pick type C disease. Traffic. 2010 Apr;11(4):419-28.

11. Appelqvist H, Sandin L, Bjornstrom K, et al. Sensitivity to lysosome-dependent cell death is directly regulated by lysosomal cholesterol content. PLoS One. 2012;7(11):e50262.

12. Nixon RA. The role of autophagy in neurodegenerative disease. Nat Med. 2013 Aug;19(8):983-97.

13. Terasawa $\mathrm{K}$, Tomabechi $\mathrm{Y}$, Ikeda $\mathrm{M}$, et al. Lysosome-associated membrane proteins-1 and -2 (LAMP-1 and LAMP-2) assemble via distinct modes. Biochemical and biophysical research communications. 2016 Oct 21;479(3):489-495.

14. Wilke S, Krausze J, Bussow K. Crystal structure of the conserved domain of the DC lysosomal associated membrane protein: implications for the lysosomal glycocalyx. BMC Biol. 2012 Jul 19;10:62.

15. Bonifacino JS, Traub LM. Signals for sorting of transmembrane proteins to endosomes and lysosomes. Annu Rev Biochem. 2003;72:395-447.

16. Braulke T, Bonifacino JS. Sorting of lysosomal proteins. Biochim Biophys Acta. 2009 Apr;1793(4):605-14.

17. Rohrer J, Schweizer A, Russell D, et al. The targeting of Lamp1 to lysosomes is dependent on the spacing of its cytoplasmic tail tyrosine sorting motif relative to the membrane. J Cell Biol. 1996 Feb;132(4):565-76.

18. Eskelinen EL. Roles of LAMP-1 and LAMP-2 in lysosome biogenesis and autophagy [Review]. Molecular aspects of medicine. 2006 Oct-Dec;27(5-6):495-502.

19. Hunziker W, Simmen T, Honing S. Trafficking of lysosomal membrane proteins in polarized kidney cells. Nephrologie. 1996;17(7):347-50.

20. Andrejewski N, Punnonen EL, Guhde G, et al. Normal lysosomal morphology and function in LAMP-1-deficient mice. J Biol Chem. 1999 Apr 30;274(18):12692-701.

21. Eskelinen EL, Illert AL, Tanaka Y, et al. Role of LAMP-2 in lysosome biogenesis and autophagy. Mol Biol Cell. 2002 Sep;13(9):3355-68.

22. Tanaka Y, Guhde G, Suter A, et al. Accumulation of autophagic vacuoles and cardiomyopathy in LAMP-2-deficient mice. Nature. 2000 Aug 24;406(6798):902-6. 
23. Nishino I, Fu J, Tanji K, et al. Primary LAMP-2 deficiency causes X-linked vacuolar cardiomyopathy and myopathy (Danon disease). Nature. 2000 Aug 24;406(6798):90610.

24. Rowland TJ, Sweet ME, Mestroni L, et al. Danon disease - dysregulation of autophagy in a multisystem disorder with cardiomyopathy. J Cell Sci. 2016 Jun 1;129(11):2135-43.

25. Hubert V, Peschel A, Langer B, et al. LAMP-2 is required for incorporating syntaxin-17 into autophagosomes and for their fusion with lysosomes. Biol Open. 2016 Oct 15;5(10):1516-1529.

26. Eskelinen EL, Schmidt CK, Neu S, et al. Disturbed cholesterol traffic but normal proteolytic function in LAMP-1/LAMP-2 double-deficient fibroblasts. Mol Biol Cell. 2004 Jul;15(7):3132-45.

27. Cuervo AM, Dice JF. A receptor for the selective uptake and degradation of proteins by lysosomes [Research Support, Non-U.S. Gov't

Research Support, U.S. Gov't, P.H.S.]. Science. 1996 Jul 26;273(5274):501-3.

28. Kaushik S, Cuervo AM. The coming of age of chaperone-mediated autophagy. Nat Rev Mol Cell Biol. 2018 Jun;19(6):365-381.

29. Scrivo A, Bourdenx $M$, Pampliega $O$, et al. Selective autophagy as a potential therapeutic target for neurodegenerative disorders. Lancet Neurol. 2018 Sep;17(9):802815.

30. Gonzalez-Polo RA, Boya P, Pauleau AL, et al. The apoptosis/autophagy paradox: autophagic vacuolization before apoptotic death. J Cell Sci. 2005 Jul 15;118(Pt 14):3091-102.

31. Chi C, Leonard A, Knight WE, et al. LAMP-2B regulates human cardiomyocyte function by mediating autophagosome-lysosome fusion. Proc Natl Acad Sci U S A. 2019 Jan 8;116(2):556-565.

32. Thurmond J, Goodman JL, Strelets VB, et al. FlyBase 2.0: the next generation. Nucleic Acids Res. 2019 Jan 8;47(D1):D759-D765.

33. Lescat L, Veron V, Mourot B, et al. Chaperone-Mediated Autophagy in the Light of Evolution: Insight from Fish. Mol Biol Evol. 2020 Oct 1;37(10):2887-2899.

34. Larkin MA, Blackshields G, Brown NP, et al. Clustal W and Clustal X version 2.0. Bioinformatics. 2007 Nov 1;23(21):2947-8.

35. Entchev EV, Schwabedissen A, Gonzalez-Gaitan M. Gradient formation of the TGF-beta homolog Dpp. Cell. 2000 Dec 8;103(6):981-91.

36. Rusten TE, Vaccari T, Lindmo K, et al. ESCRTs and Fab1 regulate distinct steps of autophagy [Research Support, N.I.H., Extramural

Research Support, Non-U.S. Gov't]. Curr Biol. 2007 Oct 23;17(20):1817-25.

37. Dunn KW, Kamocka MM, McDonald JH. A practical guide to evaluating colocalization in biological microscopy. Am J Physiol Cell Physiol. 2011 Apr;300(4):C723-42.

38. Port F, Chen HM, Lee T, et al. Optimized CRISPR/Cas tools for efficient germline and somatic genome engineering in Drosophila. Proc Natl Acad Sci U S A. 2014 Jul 22;111(29):E2967-76.

39. Tarentino AL, Plummer TH, Jr., Maley F. The release of intact oligosaccharides from specific glycoproteins by endo-beta- $\mathrm{N}$-acetylglucosaminidase $\mathrm{H}$. J Biol Chem. 1974 Feb 10;249(3):818-24.

40. Tarentino AL, Trimble RB, Plummer TH, Jr. Enzymatic approaches for studying the structure, synthesis, and processing of glycoproteins. Methods Cell Biol. 1989;32:11139.

41. Juhasz G, Neufeld TP. Experimental control and characterization of autophagy in Drosophila. Methods Mol Biol. 2008;445:125-33.

42. Ambrosio L, Morriss S, Riaz A, et al. Phylogenetic analyses and characterization of RNase X25 from Drosophila melanogaster suggest a conserved housekeeping role and 
additional functions for RNase T2 enzymes in protostomes. PLoS One. 2014;9(8):e105444.

43. Li HM, Sun L, Mittapalli O, et al. Transcriptional signatures in response to wheat germ agglutinin and starvation in Drosophila melanogaster larval midgut. Insect Mol Biol. 2009 Feb;18(1):21-31.

44. Hegedus K, Takats S, Boda A, et al. The Ccz1-Mon1-Rab7 module and Rab5 control distinct steps of autophagy. Mol Biol Cell. 2016 Oct 15;27(20):3132-3142.

45. Van Noorden CJ, Jonges TG, Van Marle J, et al. Heterogeneous suppression of experimentally induced colon cancer metastasis in rat liver lobes by inhibition of extracellular cathepsin B. Clin Exp Metastasis. 1998 Feb;16(2):159-67.

46. Honsi TG, Stenersen J. Activity and localisation of the lysosomal marker enzymes acid phosphatase, N-acetyl-beta-D-glucosaminidase, and beta-galactosidase in the earthworms Eisenia fetida and E. veneta. Comp Biochem Physiol B Biochem Mol Biol. 2000 Mar;125(3):429-37.

47. Ashtari N, Jiao X, Rahimi-Balaei M, et al. Lysosomal Acid Phosphatase Biosynthesis and Dysfunction: A Mini Review Focused on Lysosomal Enzyme Dysfunction in Brain. Curr Mol Med. 2016;16(5):439-46.

48. Rusten TE, Lindmo K, Juhasz G, et al. Programmed autophagy in the Drosophila fat body is induced by ecdysone through regulation of the PI3K pathway [Research Support, Non-U.S. Gov't]. Dev Cell. 2004 Aug;7(2):179-92.

49. Scott RC, Schuldiner O, Neufeld TP. Role and regulation of starvation-induced autophagy in the Drosophila fat body [Research Support, U.S. Gov't, P.H.S.]. Dev Cell. 2004 Aug;7(2):167-78.

50. Jacomin AC, Nezis IP. Using Fluorescent Reporters to Monitor Autophagy in the Female Germline Cells in Drosophila melanogaster. Methods Mol Biol. 2016;1457:69-78.

51. Morelli E, Ginefra P, Mastrodonato V, et al. Multiple functions of the SNARE protein Snap29 in autophagy, endocytic, and exocytic trafficking during epithelial formation in Drosophila. Autophagy. 2014;10(12):2251-68.

52. Takats S, Nagy P, Varga A, et al. Autophagosomal Syntaxin17-dependent lysosomal degradation maintains neuronal function in Drosophila. J Cell Biol. 2013 May 13;201(4):531-9.

53. Mukherjee A, Patel $B$, Koga $H$, et al. Selective endosomal microautophagy is starvationinducible in Drosophila. Autophagy. 2016 Aug 3:1984-1999.

54. Uytterhoeven V, Lauwers E, Maes I, et al. Hsc70-4 Deforms Membranes to Promote Synaptic Protein Turnover by Endosomal Microautophagy. Neuron. 2015 Nov 18;88(4):735-48.

55. Eskelinen EL, Cuervo AM, Taylor MR, et al. Unifying nomenclature for the isoforms of the lysosomal membrane protein LAMP-2. Traffic. 2005 Nov;6(11):1058-61.

56. Kaushik S, Bandyopadhyay U, Sridhar S, et al. Chaperone-mediated autophagy at a glance [Research Support, N.I.H., Extramural

Research Support, Non-U.S. Gov't]. J Cell Sci. 2011 Feb 15;124(Pt 4):495-9.

57. Koga $\mathrm{H}$, Martinez-Vicente M, Macian F, et al. A photoconvertible fluorescent reporter to track chaperone-mediated autophagy [Research Support, N.I.H., Extramural

Research Support, Non-U.S. Gov't]. Nat Commun. 2011;2:386.

58. Li J, Pfeffer SR. Lysosomal membrane glycoproteins bind cholesterol and contribute to lysosomal cholesterol export. Elife. 2016 Sep 24;5.

59. Heier C, Kuhnlein RP. Triacylglycerol Metabolism in Drosophila melanogaster. Genetics. 2018 Dec;210(4):1163-1184.

60. Xu H, Ren D. Lysosomal physiology. Annual review of physiology. 2015;77:57-80. 
61. Cheng XT, Xie YX, Zhou B, et al. Characterization of LAMP1-labeled nondegradative lysosomal and endocytic compartments in neurons. J Cell Biol. 2018 Sep 3;217(9):31273139.

62. Hu YB, Dammer EB, Ren RJ, et al. The endosomal-lysosomal system: from acidification and cargo sorting to neurodegeneration. Transl Neurodegener. 2015;4:18.

63. Huynh KK, Eskelinen EL, Scott CC, et al. LAMP proteins are required for fusion of lysosomes with phagosomes. The EMBO journal. 2007 Jan 24;26(2):313-24.

64. Sahu R, Kaushik S, Clement CC, et al. Microautophagy of cytosolic proteins by late endosomes [Research Support, N.I.H., Extramural]. Dev Cell. 2011 Jan 18;20(1):131-9.

65. Santambrogio L, Cuervo AM. Chasing the elusive mammalian microautophagy. Autophagy. 2011 Jun;7(6):652-4.

66. Meng Y, Heybrock S, Neculai D, et al. Cholesterol Handling in Lysosomes and Beyond. Trends Cell Biol. 2020 Jun;30(6):452-466.

67. Schneede A, Schmidt CK, Holtta-Vuori M, et al. Role for LAMP-2 in endosomal cholesterol transport. J Cell Mol Med. 2011 Feb;15(2):280-95.

68. Knittelfelder O, Prince E, Sales S, et al. Sterols as dietary markers for Drosophila melanogaster. Biochim Biophys Acta Mol Cell Biol Lipids. 2020 Jul;1865(7):158683.

69. Toprak U, Hegedus D, Dogan C, et al. A journey into the world of insect lipid metabolism. Arch Insect Biochem Physiol. 2020 Jun;104(2):e21682.

70. Palm W, Sampaio JL, Brankatschk M, et al. Lipoproteins in Drosophila melanogaster-assembly, function, and influence on tissue lipid composition. PLoS Genet. 2012;8(7):e1002828.

71. Matsuo N, Nagao K, Suito T, et al. Different mechanisms for selective transport of fatty acids using a single class of lipoprotein in Drosophila. J Lipid Res. 2019 Jul;60(7):11991211.

72. Couto NF, Pedersane D, Rezende L, et al. LAMP-2 absence interferes with plasma membrane repair and decreases T. cruzi host cell invasion. PLoS Negl Trop Dis. 2017 Jun;11(6):e0005657.

73. Albertti LA, Macedo AM, Chiari E, et al. Role of host lysosomal associated membrane protein (LAMP) in Trypanosoma cruzi invasion and intracellular development. Microbes Infect. 2010 Sep;12(10):784-9.

74. Petersen W, Stenzel W, Silvie O, et al. Sequestration of cholesterol within the host late endocytic pathway restricts liver-stage Plasmodium development. Mol Biol Cell. 2017 Mar 15;28(6):726-735.

75. Jacomin AC, Fauvarque MO, Taillebourg E. A functional endosomal pathway is necessary for lysosome biogenesis in Drosophila. BMC Cell Biol. 2016 Nov 16;17(1):36.

76. Thibault ST, Singer MA, Miyazaki WY, et al. A complementary transposon tool kit for Drosophila melanogaster using $P$ and piggyBac. Nature genetics. 2004 Mar;36(3):283-7.

77. Juhasz G, Hill JH, Yan Y, et al. The class III PI(3)K Vps34 promotes autophagy and endocytosis but not TOR signaling in Drosophila [Research Support, N.I.H., Extramural]. J Cell Biol. 2008 May 19;181(4):655-66.

78. Pircs K, Nagy P, Varga A, et al. Advantages and limitations of different p62-based assays for estimating autophagic activity in Drosophila. PLoS One. 2012;7(8):e44214.

79. Rao X, Huang X, Zhou Z, et al. An improvement of the $2^{\wedge}$ (-delta delta CT) method for quantitative real-time polymerase chain reaction data analysis. Biostat Bioinforma Biomath. 2013 Aug;3(3):71-85.

80. Ahmed SU, Rojo E, Kovaleva V, et al. The plant vacuolar sorting receptor AtELP is involved in transport of $\mathrm{NH}(2)$-terminal propeptide-containing vacuolar proteins in Arabidopsis thaliana. J Cell Biol. 2000 Jun 26;149(7):1335-44.

81. McVey PA, Alexander LE, Fu X, et al. Light-Dependent Changes in the Spatial Localization of Metabolites in Solenostemon scutellarioides (Coleus Henna) Visualized 
by Matrix-Free Atmospheric Pressure Electrospray Laser Desorption Ionization Mass Spectrometry Imaging. Front Plant Sci. 2018;9:1348.

82. Stein S. An integrated method for spectrum extraction and compound identification from gas chromatography/mass spectrometry data. Journal of the American Society for Mass Spectrometry. 1999;10(8):770-781.

83. Mesquita A, Glenn J, Jenny A. Differential activation of eMI by distinct forms of cellular stress. Autophagy. 2020 Jun 19.

84. Schindelin J, Arganda-Carreras I, Frise E, et al. Fiji: an open-source platform for biological-image analysis. Nat Methods. 2012 Jun 28;9(7):676-82. 


\section{Figure legends}

Figure 1. Lamp1 mutants are viable and have no developmental delay. (A) Schematic comparing Drosophila Lamp1 with human LAMP1 and LAMP2A. Note that Lamp1 has only one luminal LAMP domain. The green bar indicates the peptide region used to raise the Lamp1 antibody. (B) Schematic of Lamp1 locus with mutant alleles indicated. UTRs are in grey, coding sequence in magenta. Lamp $1^{6.1}$ contains two frame shifts $\left(^{*}\right)$ at the positions of both gRNAs used to induce it. Arrows depict location of RT-PCR primers. (C) Quantification of pupation timing shows that $\operatorname{Lamp}^{6.1}$ mutants have no developmental delay. $\mathrm{n}$ indicates total number of pupae scored. (D, E) In $3^{\text {rd }}$ instar larval fat body under fed (C) and starved conditions (D), Lamp1 colocalizes with TRITC-Dextran-labeled endolysosomes (examples marked by yellow arrow heads). Single channel images show Lamp1 (D', E') and Dextran (D”, E”), respectively. Insets show enlaged section indicated by dotted squares. Nuclei are in blue. Scale bars are 20 $\mu \mathrm{m}$ (10 $\mu \mathrm{m}$ in insets). (F) Pearson's correlation coefficient for the colocalization of Lamp1 with TRITC-Dextran under indicated conditions. As control, one channel was rotated (rot) by $180^{\circ}$. One-way ANOVAs (Tukey correction) $\mathrm{P}<0.0001$; ${ }^{* * *}, \mathrm{P}<0.0001$; ns, not significant. (G) Western blot of adult head lysates untreated (-) or treated with indicated glycosidase (+) form $w^{1118}(\mathrm{WT})$ and Lamp $1^{6.1}$ mutant flies. Blue arrowhead indicates predicted molecular mass, black arrowheads untreated Lamp1, magenta arrowheads deglycosylated forms.

Figure 2. Lamp1 mutants have strongly increased acidic structures. Compared to $w^{1118}(\mathrm{~A})$, fed $3^{\text {rd }}$ instar FB of $L a m p 1^{e 879}$ (B) $\operatorname{Lamp} 1^{11 B}$ (C) show a strong increase of LTR staining, a phenotype that is rescued by a duplication including Lamp1 (D; quantified in I). (E, F) LTR staining is also increased in starved $3^{\text {rd }}$ instar FB of Lamp $1^{\text {e879 }}$ mutants (compare E, F; quantified in I). (G, H) A similar increase in the acidic compartment is seen in adult fat body of Lamp $1^{e 879}$ (compare G, H; quantified in J). Nuclei are in blue; greyscale images show LTR channel; scale bars: $20 \mu \mathrm{m}$. (I, J) Quantification LTR staining in larval (I) and adult (J) fat body of indicated genotypes. One-way ANOVA (Tukey correction) for Fed in (I) $\mathrm{P}<0.0001$; T-tests (J); ${ }^{* *} \mathrm{P}<0.01,{ }^{* * *}, \mathrm{P}<0.0001$; ns, not significant. (K) Amyrel is normally induced upon starvation in Lamp $1^{e 879}$ mutant larvae (qPCR normalized to RpL32). Two-tailed T-test.

Figure 3. Lysosomal activity. (A-C) Cathepsin B activity as measured by Magic Red assay is not significantly increased in FB of fed $3^{\text {rd }}$ instar larvae (compare $w^{1118}$ in A with Lamp $1^{6.1}$ (B) and $L a m p 1^{11 B}(\mathrm{C})$; quantified in $\left.\mathrm{G}\right)$. In contrast, under starved conditions (D-F), Lamp $1^{6.1}(\mathrm{E})$ and Lamp $1^{11 B}(\mathrm{~F})$ have increased lysosomal activity (compare to $w^{1118}$ in $\mathrm{D}$; quantified in $\mathrm{G}$ ). Nuclei 
are in blue; greyscale images show Magic Red channel; scale bar: $20 \mu \mathrm{m}$. (G) Quantification of Magic Red puncta in FB of indicated genotypes. One-way ANOVA (Tukey correction) $\mathrm{P}<0.001$. $(\mathrm{H})$ Normalized Acid Phosphatase activity of lysates enriched for lysosomes of fed $3^{\text {rd }}$ instar larvae of indicated genotypes. One-way ANOVA (Dunnett correction). * $\mathrm{P}<0.05$; ${ }^{* *} \mathrm{P}<0.01$, ns, not significant.

Figure 4. Autophagic flux is normal in FB of Lamp1 mutants. $(A, B)$ Under basal conditions, $M A$ in $3^{\text {rd }}$ instar FB is low and indistinguishable between Lamp $1^{6.1}$ heterozygous control $(A)$, Lamp1 $1^{6.1} / \operatorname{Lamp}^{11 B}(\mathrm{~B})$, and homozygous Lamp1 $1^{6.1}$ (quantified in E) mutants. (C, D) MA is normally induced by starvation in Lamp $1^{6.1}$ heterozygotes (C), Lamp1 $1^{6.1} / \operatorname{Lamp} 1^{11 B}$ (D), and homozygous Lamp1 $1^{6.1}$ (quantified in E). APGs are labeled with GFP and mCherry, while GFP fluorescence is quenched by the acidic $\mathrm{pH}$ in autolysosomes, which are thus labeled in red only. (E) Quantification of GFP puncta (APGs) and cherry puncta (APGs and APGLs) shows normal MA flux in Lamp1 mutants with no difference to controls. One-way ANOVA (Tukey correction) $\mathrm{P}<0.0001$. (F, G) Snap29 (green) recruitment to APGs (marked by mCherry-Atg8a; C-Atg8a; red) is normal in FB of $\operatorname{Lamp} 1^{6.1} / \operatorname{Lamp} 1^{11 B}$ transheterozygotes $(\mathrm{G})$ and $\operatorname{Lamp} 1^{6.1}$ mutants (quantified in $\mathrm{H}$; compare to heterozygotes in $\mathrm{F}$; examples of APGs are marked by yellow arrowheads). (H) Pearson's correlation coefficient of colocalization of Snap29 with mCherryAtg8a of indicated genotypes. One-way ANOVA; Dunnett correction) ns. Nuclei are in blue; greyscale images show indicated channels; scale bars: $20 \mu \mathrm{m}$. (I) Lamp $1^{\text {e879 }}$ mutant adults have normal expression of the essential MA genes Atg5 and Atg8 (fed; normalized to RpL32). Twotailed T-tests. ${ }^{\star \star *} \mathrm{P}<0.001,{ }^{\star \star * \star}, \mathrm{P}<0.0001$; ns, not significant.

Figure 5. eMl induced by prolonged starvation is normal in Lamp1 $1^{6.1}$ mutants (compare starved Lamp $1^{6.1}$ heterozygous control (A) and homozygous Lamp $1^{6.1}$ mutant larval FB (B) with corresponding fed tissue (C,D). Greyscale images show eMI sensor; nuclei are in blue; scale bar 20 $\mu \mathrm{m}$. (E) quantification of eMI sensor puncta per $3^{\text {rd }}$ instar FB cell of indicated genotypes. One-way ANOVA (Tukey correction) $\mathrm{P}<0.0001$; ** $\mathrm{P}<0.01$; ns, not significant.

Figure 6. TEM analyses of Lamp1 mutant fat body. (A-E) Examples of autolysosomal structures of Lamp $1^{6.1}$ mutant $(A-C)$ and WT (D) $3^{\text {rd }}$ instar fed larval fat body. APGL: autophagolysosome; AL: autolysosome; L: lysosome; LD: lipid droplet. See also methods for structure definitions. Scale bars: $0.5 \mu \mathrm{m}$. (F-H) Quantification of density (area per field of view) of APGLs (F), ALs $(G)$, and lysosomes $(H)$ under indicated conditions. Mann-Whitney tests. (I) 
Size distribution of APGLs under indicated conditions. Mann-Whitney and Kolmogorov-Smirnov tests. * $\mathrm{P}<0.05$; ns: not significant.

Figure 7. Lamp1 mutant larvae have altered lipid levels. (A) Sterol levels are increased in Lamp $1^{e 879}$ mutants, an effect that is partially rescued by a duplication including Lamp1 (Dp). Two-tailed T-tests. (B) Triacylglycerols (TAG) are unaffected. (C) Levels of larval DAGs and their indicated subclasses in $\operatorname{Lamp} 1^{e 879}$ mutants. Note the rescue by $L a m p 1^{D p}$. One-way ANOVA (Tukey correction) significance levels are indicated below the $\mathrm{X}$-axis. Unless indicated, changes are not significant (ns). * $\mathrm{P}<0.05 ;{ }^{* *}, \mathrm{P}<0.01$.

Figure S1. (A) Sequence of WT (upper line) and indicated Lamp1 Crispr alleles (respective lower lines). Net deletions are indicated. In $\operatorname{Lamp} 1^{6.1}$, the first of the two frame shifts results in addition of 32 non-Lamp1 aa after aa 90 (well upstream of the transmembrane domain). gRNA target sequences are indicated in blue, start/stop codons in red, and inserted bases are in green. (B) Lamp $1^{e 879}$ is an RNA null allele. Note that the duplication $\mathrm{Dp}(2 ; 3) P 6-B 2^{[L a m p 1]}$ uncovering Lamp1 (Dp) rescues expression to normal levels. RpL32 was used as positive control. (C, D) Western blots of fed (F) and $4 \mathrm{~h}$ starved (ST) adult head (C) and $3^{\text {rd }}$ instar larval (D) lysates show that Lamp1 migrates as a major form of $\sim 45 \mathrm{kDa}$ with several minor forms up to $\sim 60 \mathrm{kDa}$ (magenta arrowheads; compare WT with Lamp16.1 and Lamp1 ${ }^{11 B}$ lanes for specificity). Blue arrowheads indicate predicted molecular mass. aTubulin was used as loading control shown in lower panels. $(\mathrm{E}-\mathrm{H})$ Compared to fed and starved fat body of $w^{1118} 3^{\text {rd }}$ instar FB $(E, G), L_{1} 1^{6.1}$ mutants $(F, H)$ lack Lamp1 staining (green), demonstrating specificity of the antibody also for histochemistry. Nuclei in blue. Scale bar: $10 \mu \mathrm{m}$.

Figure S2. Increased LTR staining in $3^{\text {rd }}$ instar FB of Lamp1 mutants. (A, D) $w^{1118}$ control. $(\mathrm{B}, \mathrm{E}) \operatorname{Lamp} 1^{6.1}$ and $(\mathrm{C}, \mathrm{F})$ Lamp $1^{11 B}$ mutants. A-B: fed, D-F starved conditions. LTR in red, nuclei in blue; scale bar: $20 \mu \mathrm{m}$.

Figure S3. TEM analyses of density (area per field of view) of lipid droplets (A) and glycogen (B) in fed and starved WT and Lamp $1^{6.1}$ mutant larvae. Mann-Whitney tests; ns: not significant. 
Dm Lamp1

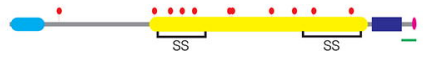

Hs LAMP1

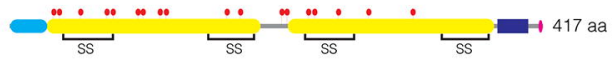

Hs LAMP2A

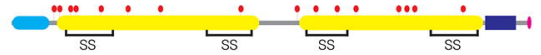

410 aa

Sig. pep.

LAMP dom.

TM, dom. SS Disulphide Bridge

I Lys. targ. motif $\bullet$ (pred.) N-Glyc.

B

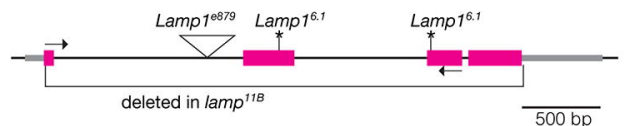

L3; fed

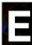

L3; st.

F

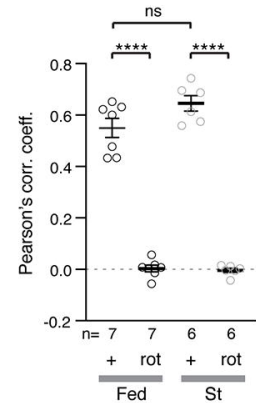

\section{$w^{1118}$ \\ D'}

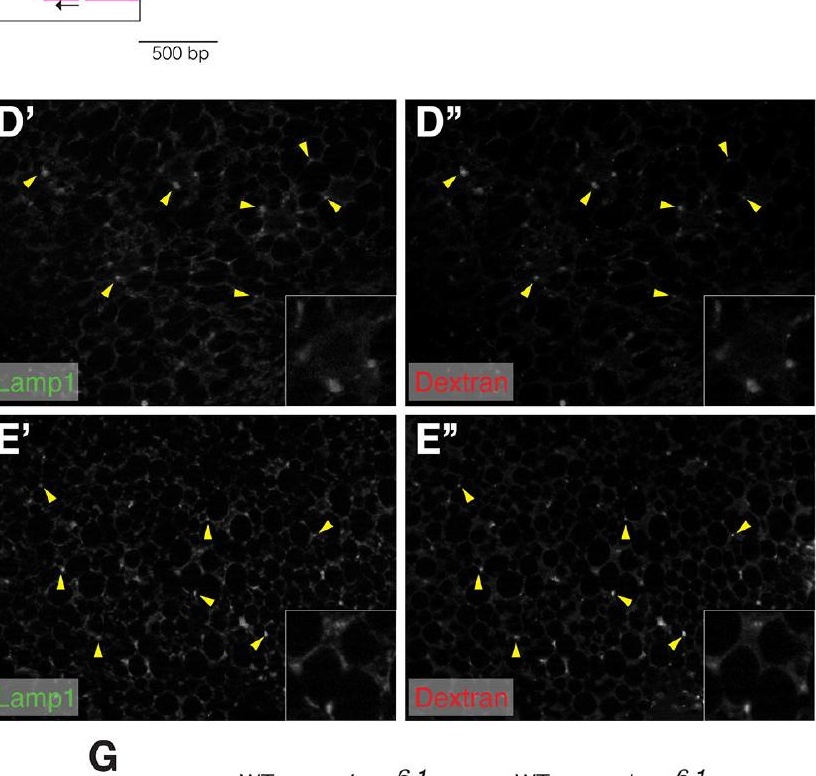

L

w1118

E

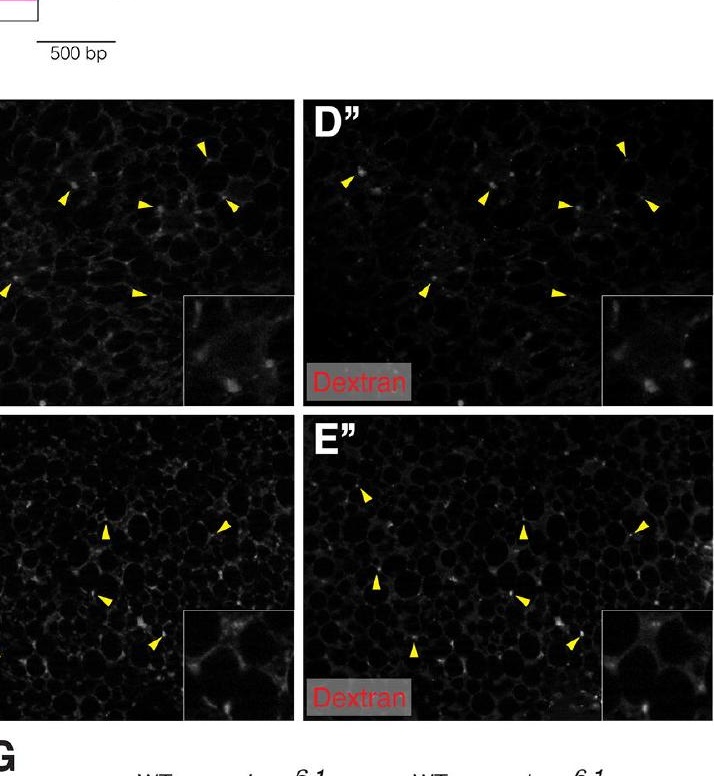

E

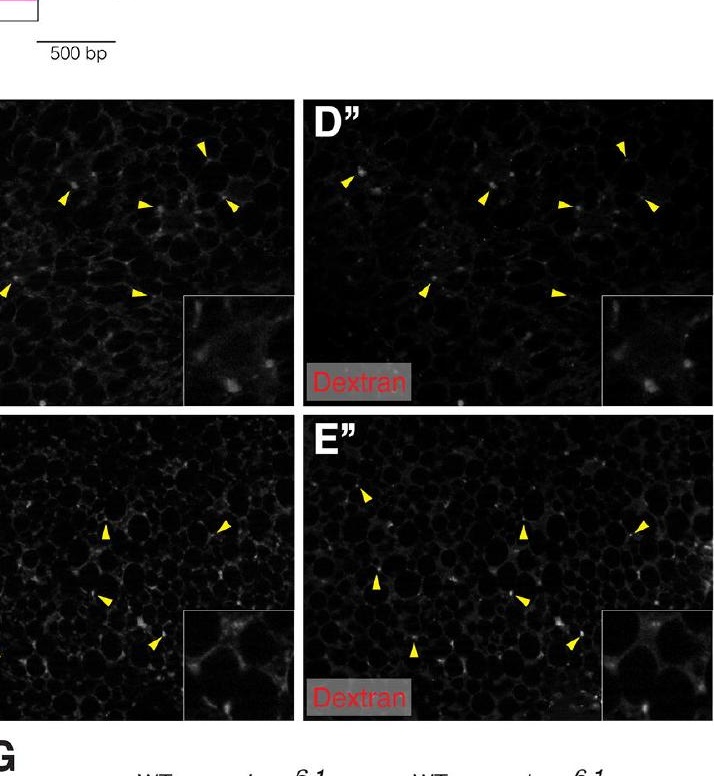

G

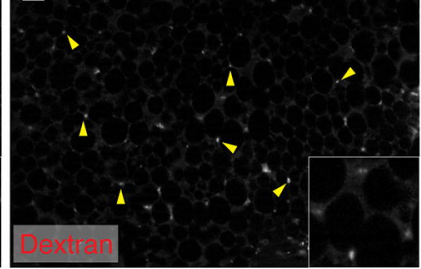

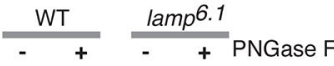

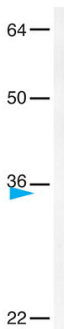

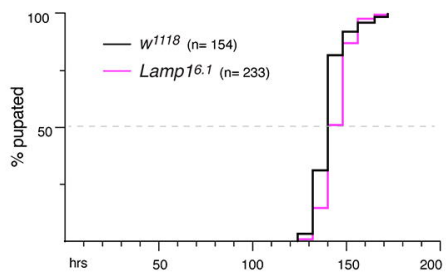

D"

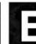




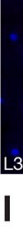

L3; fed
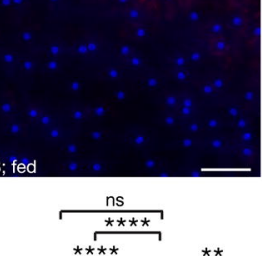

$\frac{\pi}{5}$

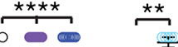

$80-$ 壬 ${ }_{0}$ 平

ㄴ. 8

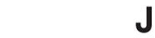

J

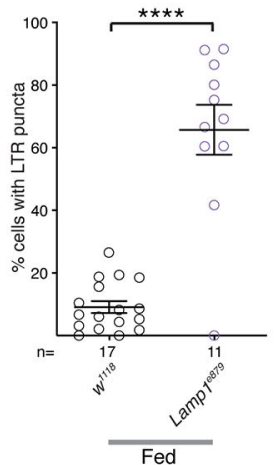

Adult; fed
K

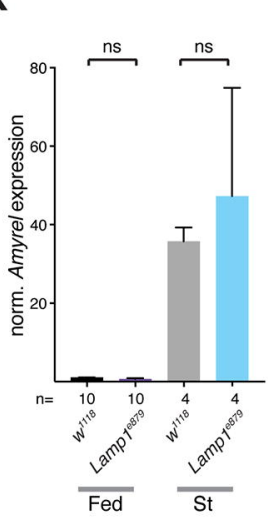

\section{Lamp1 $\left.^{\text {e879 }}\right|^{\prime}$}

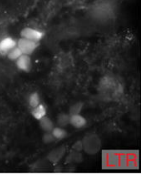



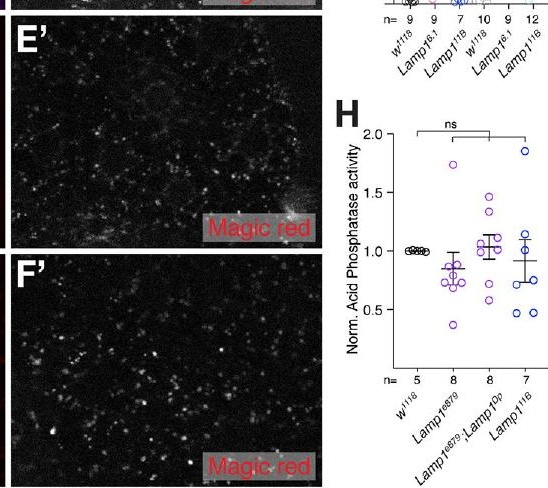

\section{L3; starved}

E

L3; starved

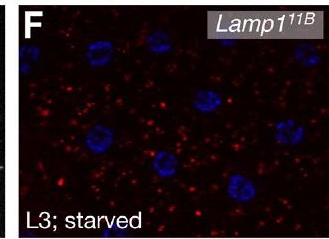




\section{Starved}

\section{Lamp16.1/Lamp111B}

$\mathbf{F}$

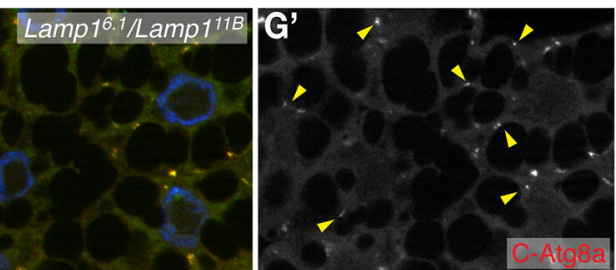

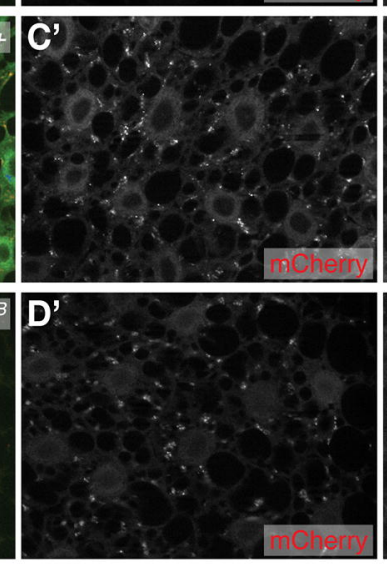
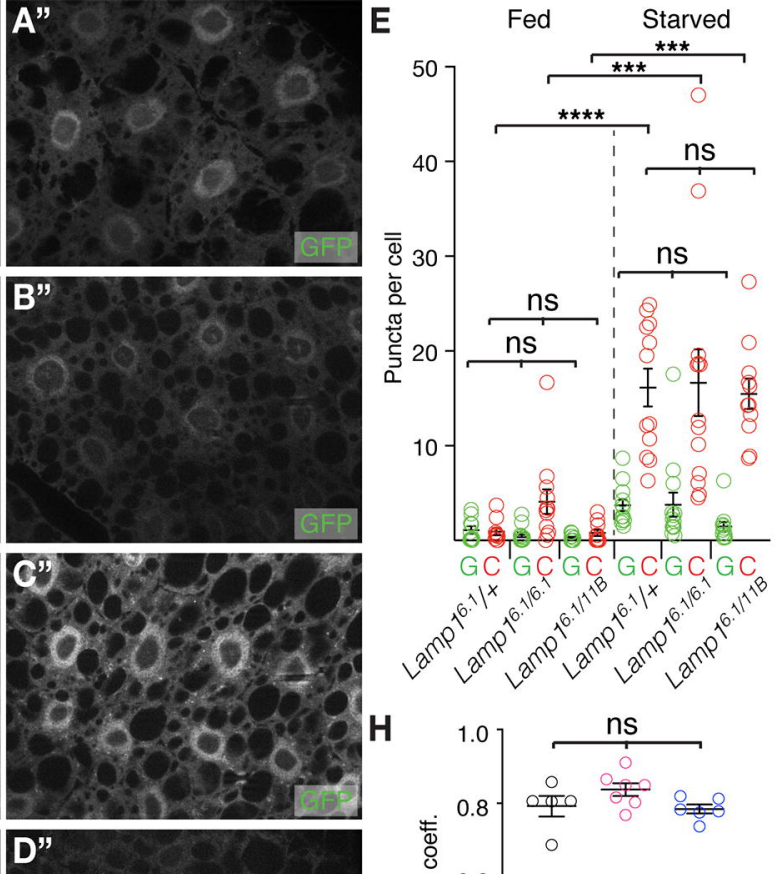

GC GC GC GCGC GC

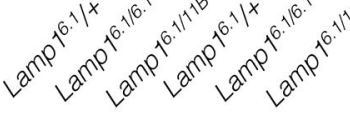

H

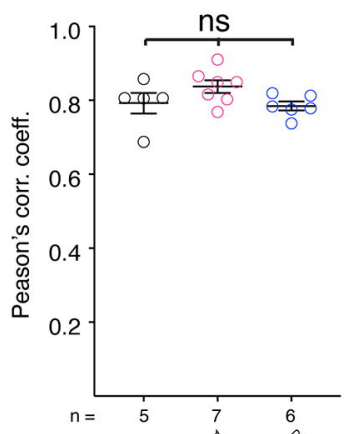

$F$

GEP
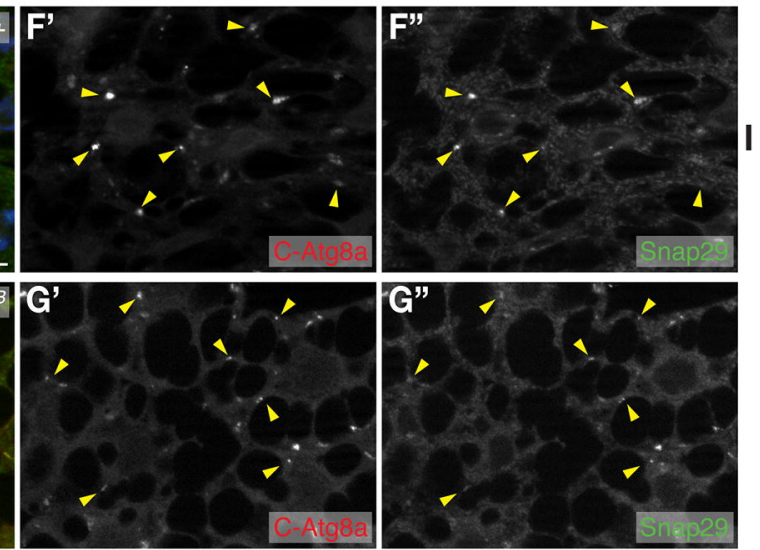
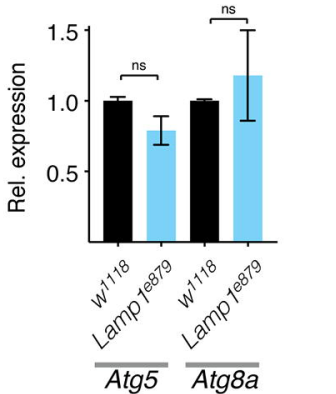


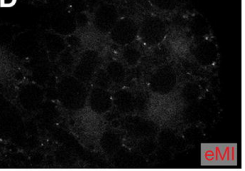



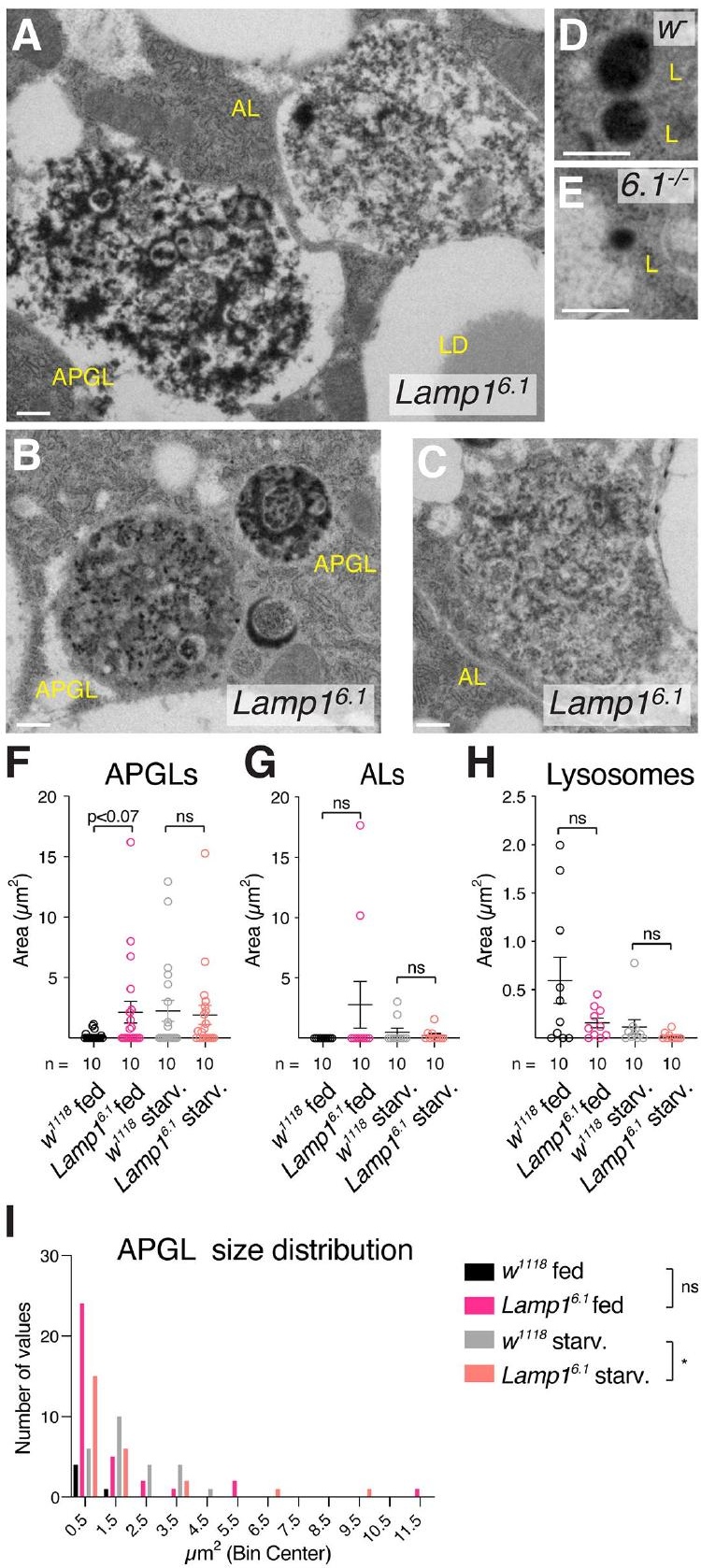

F
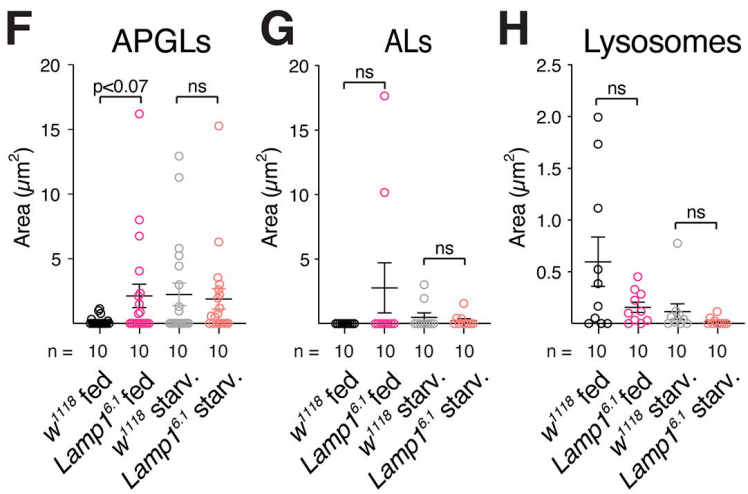
$\mu \mathrm{m}^{2}$ (Bin Center) 

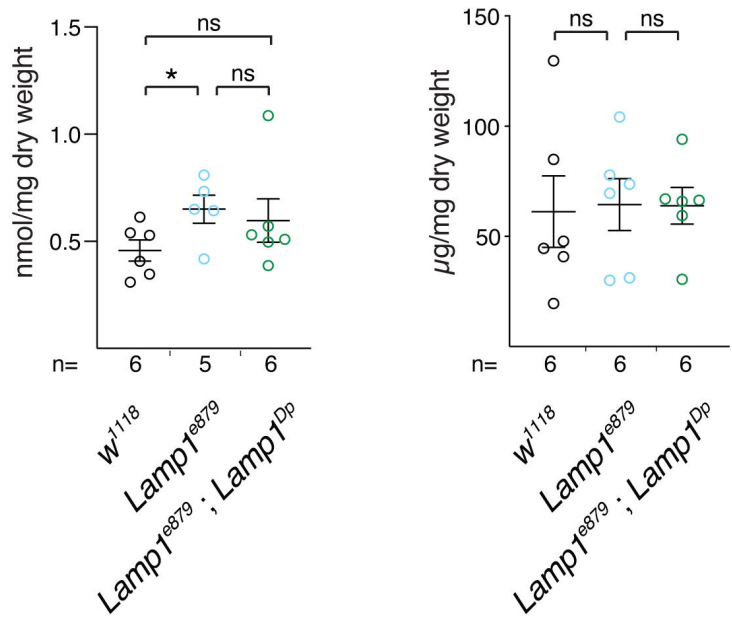

\section{DAGs}

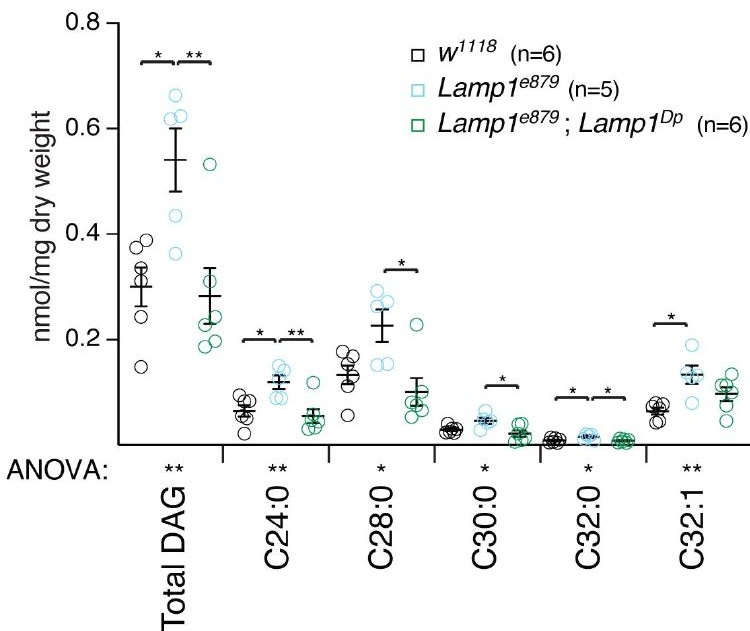

